\title{
Inverse statistics of smooth signals: the case of two dimensional turbulence
}

\author{
L. Biferale ${ }^{1}$, M. Cencini ${ }^{2}$, A. Lanotte ${ }^{1}$, D. Vergni ${ }^{2}$ and A. Vulpiani ${ }^{2}$ \\ ${ }^{1}$ Dipartimento di Fisica and INFM , Università "Tor Vergata", Via della Ricerca Scientifica 1, I-00133 Roma, Italy \\ ${ }^{2}$ Dipartimento di Fisica and INFM , Università "La Sapienza", Piazza Aldo Moro 2, I-00185 Roma, Italy
}

The problem of inverse statistics (statistics of distances for which the signal fluctuations are larger than a certain threshold) in differentiable signals with power law spectrum, $E(k) \sim k^{-\alpha}$, $3 \leq \alpha<5$, is discussed. We show that for these signals, with random phases, exit-distance moments follow a bi-fractal distribution. We also investigate two dimensional turbulent flows in the direct cascade regime, which display a more complex behavior. We give numerical evidences that the inverse statistics of $2 \mathrm{~d}$ turbulent flows is described by a multi-fractal probability distribution, i.e. the statistics of laminar events is not simply captured by the exponent $\alpha$ characterizing the spectrum. PACS number(s) : 47.27.Eq, 05.45.-a

Many phenomena in natural science posses complex behaviors over a wide range of spatial and temporal scales. Complexity is quantified by the non-Gaussian properties of probability distribution functions (pdf) of signal increments over a given range of scales. Whenever pdf's at different scales cannot be superposed by a simple rescaling procedure one speaks about intermittency [1]. Such non-trivial rescaling properties may be exhibited by pdf's tails or peaks, or both [2]. Strongly intermittent and rough signals, like those encountered in three dimensional turbulent flows, are the typical examples of systems with non-trivial, say multiaffine, scaling of pdf's tails. Other important natural phenomena develop simple pdf's tails but non-trivial pdf's peaks. This is the case of two dimensional turbulence as it will be shown in this letter. Indeed laminar fluctuations, corresponding to the events described by the peak of the probability distribution, posses non-trivial scaling properties. Recently, it has been shown that laminar fluctuations of rough and multiaffine fields are optimally characterized in terms of their exit-distance statistics, also known as inverse-statistics 3 [6].

The aim of this letter is twofold. First we want to extend the application of inverse statistics [3.4] to the case of smooth signals with a given power spectrum, $E(k) \sim k^{-\alpha}$. In particular, we discuss signals only one time differentiable, i.e. $3 \leq \alpha<5$. For such signals, direct statistics, i.e. moments of signal increments over a given scale, do not bring any information: they are always dominated by the differentiable event, $v(x+r)-v(x) \sim r$, which are always present when the spectrum has a slope $\alpha>3$. On the contrary, we will show that the exit-distance statistics is given by a bifractal distribution if only one kind of more than smooth fluctuation exists. With more than smooth fluctuations we mean events where the signal has a local scaling as $v(x+r)-v(x) \sim r^{h}$ with $h>1$.

Second, we will apply the inverse statistics analysis to the case of two dimensional turbulence. Two-dimensional turbulence in the direct enstrophy cascade regime is of obvious importance both theoretically and practically to understand a variety of different natural processes, e.g. in geophysics and astrophysics [7, 8 . By applying the exitdistance analysis to a set of $2 \mathrm{~d}$ numerical simulations, we will show in a quantitative way that laminar events of two-dimensional turbulence posses highly non-trivial rescaling properties, revealing a rich (multifractal) structure of laminar fluctuations. We also discuss the importance of large-scale structures in determining the inverse statistics of two dimensional turbulent flows.

Let us begin by considering a one dimensional signal built by fixing its spectrum as $E(k) \sim k^{-\alpha}$ on all available wave-numbers :

$$
v(x)=\sum_{k} \hat{v}(k) e^{i\left(x k+\theta_{k}\right)},
$$

with $|\hat{v}(k)|^{2} \sim k^{-\alpha}$ and $\theta_{k}$ random phases, uniformly distributed in $[0,2 \pi]$. If $3 \leq \alpha<5$ the signal is everywhere one-time differentiable : it follows that moments of its difference over any increment $r$ always have a differentiable scaling, namely

$$
S_{p}(r)=\left\langle(v(x+r)-v(x))^{p}\right\rangle \sim c_{p} r^{p},
$$

while moments with $p \leq-1$ do not exist. In order to highlight the role of non-trivial more than smooth stochastic fluctuations associated to the spectrum slope $\alpha$, one needs observable sensitive to laminar fluctuations like moments of inverse statistics. With inverse statistics we mean moments of increments, $r(\delta v)$, necessary to observe in the signal a forward (backward) exit through a barrier $\delta v$. In particular, we fix the height of the barrier, $\delta v$, and we pick at random a reference point $x_{0}$. Then, we measure the first forward or backward exit, $r(\delta v)$, i.e. the first point such that $\left|v\left(x_{0} \pm r\right)-v\left(x_{0}\right)\right| \geq \delta v$ and we repeat the observations for many $x_{0}$ and for different barrier heights. This allows us to define the probability distribution for exit events, $\mathcal{P}(r(\delta v))$.

Let us define, the exit moments through a barrier $\delta v$ as:

$$
T^{(p)}(\delta v)=\left\langle r^{p}(\delta v)\right\rangle,
$$

where the average is taken with respect to the random choice of $x_{0}$ [9]. Positive moments of exit events preferentially weight smooth, laminar, fluctuations. Exit-event 
moments are also called inverse statistics moments because of the difference with direct statistics where one measure signal increments over a given scale. For the prototype smooth signal (11), a rigorous estimate of the scaling exponents of inverse statistics moments can be derived as follows. From (1), when the spectrum exponent is in the window of first order differentiability, $3 \leq \alpha<5$, we may estimate the typical fluctuations as

$$
v\left(x_{0}+r\right)-v\left(x_{0}\right) \sim \partial_{x_{0}} v\left(x_{0}\right) \boldsymbol{r}+c\left(x_{0}\right) r^{h},
$$

where we have kept only the two most important scaling behaviors: $O(r)$ because of the differentiability and $O\left(r^{h}\right)$ from the spectrum exponent. In (3), the exponent $1 \leq h<2$ is connected to the spectrum slope by the dimensional relation $\alpha=2 h+1$, while the function $c(x)$ is a continuous function of $x$. By studying the exit event, in the limit of small barrier height, we select with probability one the differentiable scaling $r(\delta v) \sim \delta v$ except for those $x_{0}$ s where the first derivative, $\partial_{x} v\left(x_{0}\right)$, vanishes. In the latter case, the $O\left(r^{h}\right)$ term dominates the scaling behavior. With $3 \leq \alpha<5$ the first derivative is a self-affine signal with Hölder exponent $\xi=h-1$, i.e. $\partial_{x} v(x+r)-\partial_{x} v(x) \sim r^{\xi}$, that is it vanishes on a fractal set of dimension $D=1-\xi=2-h$. Therefore, the probability to see the sub-dominant term $O\left(r^{h}\right)$ dominating the exit events in (3) is given by the probability to pick a point at random on a fractal set with dimension $D$, i.e.

$$
P\left(r \sim(\delta v)^{1 / h}\right) \sim r^{1-D}=(\delta v)^{1-1 / h} .
$$

Taking into account both situations, we end with the following bi-fractal prediction for inverse statistics moments :

$$
T^{(p)}(\delta v) \sim \delta v^{\chi_{\mathrm{bf}}(p)}, \chi_{\mathrm{bf}}(p)=\min \left(p, \frac{p}{h}+1-\frac{1}{h}\right) .
$$

From the previous bi-fractal formula, one sees that laminar, differentiable, fluctuations influence the inverse statistics only up to moments of order $p=1$; for larger $p$, the pdf is dominated by the sub-dominant behavior, $v(x+r)-v(x) \sim r^{h}$. In other words, the extrema of the signal play the role of singularities in inverse statistics: close to the extrema, events with much longer exits through barriers of order $\delta v$ are observed when $\delta v \rightarrow 0$. In Fig. 1, we numerically check this prediction on a one-dimensional signal with $\alpha=4$, i.e. with $h=1.5$. The derivative of such a signal has an Hölder exponent $\xi=0.5$, i.e. it is a stationary Brownian motion. As shown, the prediction is verified with high accuracy.

Switching to real signals, we consider two dimensional incompressible turbulent flows. As it is well known, 2d turbulence is characterized by a forward enstrophy cascade from forced scales to dissipative ones [8]. In the inertial range, arguments á la Kolmogorov give for the velocity spectrum the prediction $E(k) \sim k^{-3}$ plus logarithmic corrections [8], which is experimentally [10] and numerically [11] observed. However, it is also measured a more than smooth spectrum $E(k) \sim k^{-\alpha}$ with $\alpha>3$ depending on the characteristics of the forcing and large-scale dissipation [12,13. The dependency of inertial range statistics from large-scale effects (universality issue) is still an open problem in $2 \mathrm{~d}$ turbulence in the direct cascade regime.

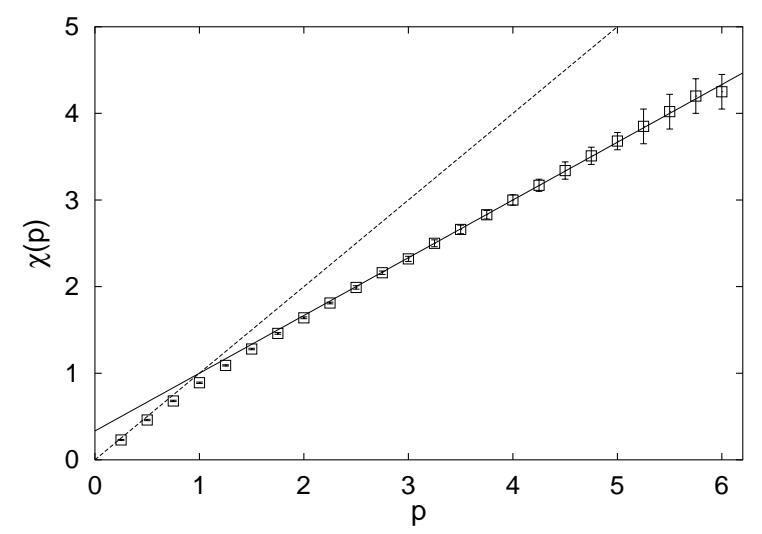

FIG. 1. Scaling exponents $\chi(p)$ for the 1-d signal (11) with $\alpha=4$. The dashed line refers to the linear differentiable behavior for $p \leq 1, \chi_{\mathrm{bf}}(p)=p$. The continuous line gives the more than smooth behavior, $\chi_{\mathrm{bf}}(p)=(p / h+1-1 / h)$. Moments (2) have been computed using $10^{3}$ realizations of the signal (11) with $2^{17}$ modes; for each realization $2^{12}$ starting points, $x_{0}$, have been taken at random.

The most important feature of the vorticity cascade is the presence of a strong interaction between eddies of very different scales: such a non-locality (in Fourier space) should play a fundamental role in shaping the energy spectrum, in particular the strong dependency of the spectrum slope from large-scale effects. In order to understand the importance of large-scale statistics one needs to go beyond the spectrum slope.

We analyze exit-distance statistics in a series of direct numerical simulations of the two-dimensional NavierStokes equation:

$$
\partial_{t} \omega+J(\omega, \psi)=\nu \Delta^{q} \omega-\beta_{\rho} \Delta^{-\rho} \omega+F,
$$

where $\omega$ is the vorticity, $\psi$ the stream function, and $J$ the Jacobian. We use a standard dealiased pseudo-spectral algorithm with periodic boundary conditions, at resolutions $512^{2}$ and $1024^{2}$. The large-scale forcing $\mathrm{F}$ is Gaussian, white-in-time, and nonzero only at some characteristic wave-numbers $k_{f}$ between 4 and 6 . Enstrophy is dissipated at small scales with an hyper-viscosity $q=4$. Energy is removed at large scales to avoid piling up on the smallest mode. We performed two sets of numerical simulations with different IR draining, with an inverse Laplacian with $\rho=2$ case (A) and with $\rho=0$ case (B), without observing big differences in the spectrum slope. In Fig. 2 (inset) we show the compensated average spec- 
trum that we observe for case (A): notice the strong influence of coherent structures for low wave-numbers. The best fit to the spectrum slope for this case gives $\alpha=3.26 \pm 0.06$, i.e. a more than smooth exponent $h=1.13$, while for the case (B) we found $\alpha=3.24 \pm 0.06$. The relevant test we wish to perform on the $2 \mathrm{~d}$ flow is comparing the inverse statistics measured on several snapshots of the direct numerical simulations with the inverse statistics obtained after randomization of all velocity phases on the same frames. We measured the moments of exit events using both transversal and longitudinal velocity increments because they could reflect in a different way the presence of coherent structures.

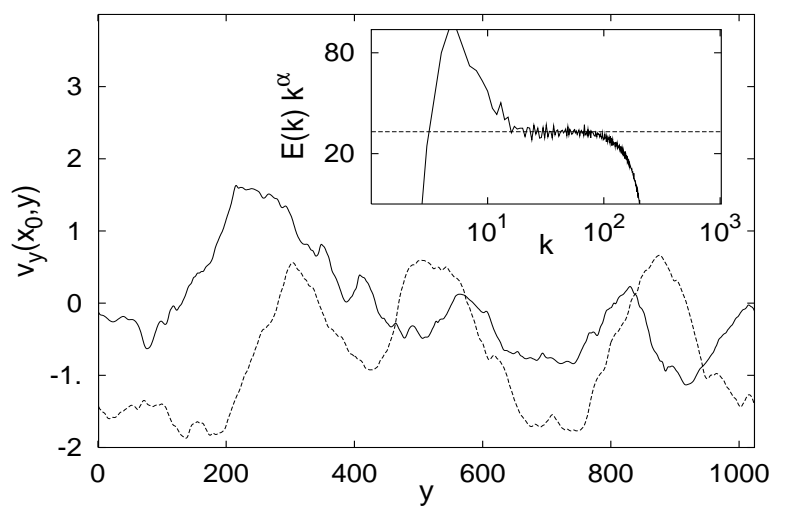

FIG. 2. One dimensional longitudinal cut of the velocity field $v_{y}\left(x_{0}, y\right)$ (continuous line); the same after randomization of the phases (dashed line). In the inset the averaged compensated spectrum, with the best fit in the inertial range $\alpha=3.26 \pm 0.06$

The rationale for this test is to investigate the importance of correlations between fluctuations at different wave-numbers and therefore the "information" content brought by coherent structures in $2 \mathrm{~d}$ turbulent flows. In Fig. 2, a one-dimensional cut of the 2 -d velocity field before and after phases randomization is plotted. At a first glance, it is rather difficult to distinguish between the true dynamical and the randomized field. This is due to the steepness of the spectrum, i.e. only few modes dominate the real-space configuration. Despite the apparent similarity, there are big statistical differences between the two fields. Looking at inverse moments we measure a clear departure of the true turbulent statistics from the bi-fractal prediction (5), while the randomized configurations are in agreement with it, with $h=1.13$. Due to the numerical limited resolution, in order to perform a quantitative statement, one can evaluate only relative scaling properties. Therefore, we measure scaling laws of the inverse statistics by plotting all moments $T^{(p)}(\delta v)$ versus a reference one, say $T^{(2)}(\delta v)$. This is the same technique called ESS [14] fruitfully applied in the direct analysis of $3 \mathrm{~d}$ turbulent data with the aim of re-adsorbing some finite size effects and extracting scaling information also at moderate resolution. Plotting data in this way allows quantitative statements only for relative exit-distance exponents, $\chi(p) / \chi(2)$.

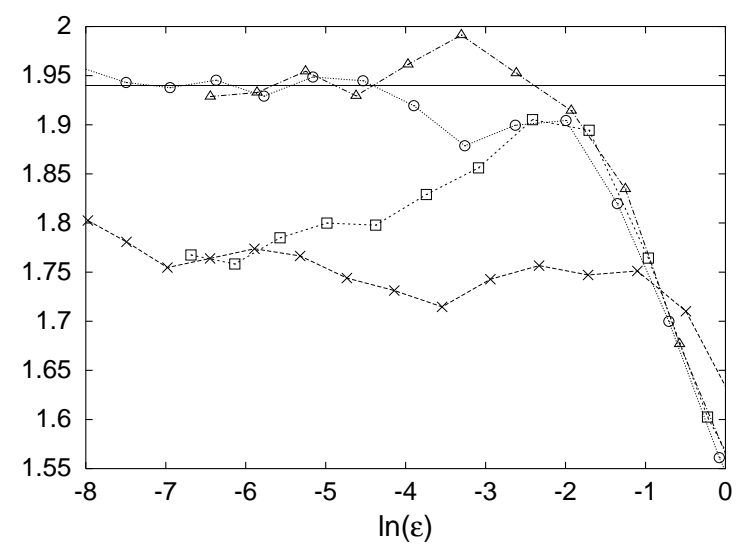

FIG. 3. Logarithmic local slopes for the relative scaling $T^{(4)}(\delta v)$ vs $T^{(2)}(\delta v)$ : for the longitudinal $(\square)$ and transverse exit-distance $(\times)$ for run $(A)$. The same quantities after the phases randomization are represented by $(\triangle)$ and (०), respectively.

In Fig. 3 we show the logarithmic local slopes for the relative scaling of $T^{(4)}(\delta v)$ vs $T^{(2)}(\delta v)$ for longitudinal and transversal velocity increments. We stress two important results. First, the inverse-statistics moment exponents measured on the fields from the numerical simulations after phases randomization coincide with the prediction (5) with $h=1.13$ as extracted from the averaged spectrum. On the other hand, the longitudinal and transversal inverse-statistics moments without phases randomization have a more complex, intermittent, distribution i.e. they are not described by the bi-fractal prediction (5). Second, longitudinal and transversal moments are slightly different, indicating that longitudinal and transversal velocity fluctuations probe differently the smooth part of the $2 \mathrm{~d}$ field. This is of course connected to the fact that longitudinal or transversal velocity differences have different profiles when measured across coherent vortical structures. We also note that transversal exit moments display a better scaling behavior than the longitudinal ones.

In Fig. 4, we summarize our results showing the curve $\chi(p) / \chi(2)$ for both randomized and not-randomized longitudinal and transversal exit moments for run (A) [15]. Notice that for $p<1$ randomized and not randomized data almost coincide because those moments are dominated by the differentiable fluctuations $v(x+r)-v(x) \sim r$ and therefore the relative scaling exponents differ only due to the factor $\chi(2)$ which is almost the same for both data sets. On the other hand, clear different statistics are measured, for $p>1$, by comparing the true dynamical longitudinal and transversal exit moments with the randomized ones. 
In conclusion, we have given an estimate of inversestatistics moments for signals with a more than smooth spectrum, i.e. signals which are differentiable and with non-trivial stochastic sub-leading fluctuations. We have also shown that statistical properties of a $2 \mathrm{~d}$ turbulent flow are not simply summarized by the spectrum slope.

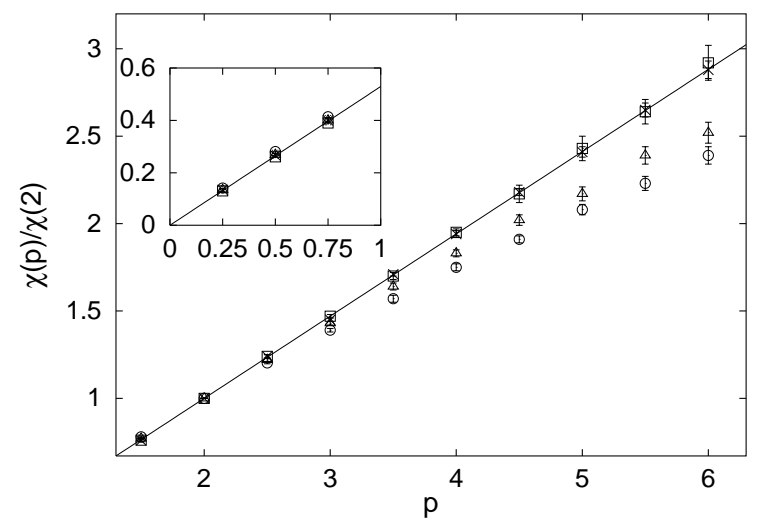

FIG. 4. $\chi(p) / \chi(2)$ for $1.5 \leq p \leq 6$ for both longitudinal $(\triangle)$ and transversal (o) exit moments obtained in run (A), $(\square)$ and $(\times)$ indicate the same after randomization, respectively. Inset: the same but for $p<1$. The continuous line is the bi-fractal prediction $\chi_{\mathrm{bf}}(p) / \chi_{\mathrm{bf}}(2)$, with $h=1$.13. Error bars have been estimated as the maximum deviation of the local slopes from the average value. Run (A) gives indistinguishable results (not shown).

From the exit-distance analysis it is possible to highlight a whole spectrum of more than smooth fluctuations. Such fluctuations, being connected with laminar events, are the strongest statistical signature of large scale coherence. Experiments with different methods of removing/pumping energy at large scales should be performed, to investigate the importance of large-scales structures in the inverse statistics of flows with different spectra. A more quantitative comprehension of the multifractality of inverse statistics could be inferred trying to connect scaling exponents to the finite time (Lagrangian) Lyapunov exponents and the drag coefficient, by extending the analysis proposed by Ott and collaborators 13. As a final remark we would like to stress that inverse statistics provide a completely new statistical indicator with respect to the standard direct statistics observable. We have shown that such method is necessary in all those cases where non-trivial fluctuations are sub-leading with respect to the differentiable contributions. Obviously, the same kind of analysis here reported can be extended to temporal signals, opening the possibility of applying the method to a broad class of natural phenomena. As an example, we just mention possible applications in situations common to climatology or meteorology where estimating the probability of persistent velocity configuration, or of any other dynamical variable, is relevant. As a perspective, one important generalization would the investigation of multi-dimensional signals by studying the statistics of d-dimensional volumes between equispaced iso-surfaces. The latter method may be, for example, important for analyzing coherence properties of two-dimensional or multi-dimensional patterns.

We acknowledge useful discussions with R. Benzi, G. Boffetta and G. Eyink. This work has been partially supported by the EU under the Grant No. HPRNCT 2000-00162 "Non Ideal Turbulence" and the Grant ERB FMR XCT 98-0175 "Intermittency in Turbulent Systems". We also acknowledge INFM support (Iniziativa di Calcolo Parallelo).

[1] U. Frisch, Turbulence: The Legacy of A.N. Kolmogorov, Cambridge University Press, Cambridge, (1995).

[2] A. Celani, A. Lanotte, A. Mazzino and M. Vergassola, Phys. Fluids 13, 1768, (2001).

[3] M. H. Jensen, Phys. Rev. Lett. 8376 (1999).

[4] L. Biferale, M. Cencini, D. Vergni, and A. Vulpiani Phys. Rev. E 60, R6295 (1999).

[5] M. Abel, L. Biferale, M. Cencini, M. Falcioni, D. Vergni, and A. Vulpiani, Phys. Rev. Lett. 84, 6002 (2000);

[6] M. Abel, L. Biferale, M. Cencini, M. Falcioni, D. Vergni, and A. Vulpiani, Physica D 147, 12 (2000).

[7] M. Lesieur, Turbulence in Fluids, 2nd ed., Kluwer Ac. Publish., London (1990).

[8] R. H. Kraichnan, Physics of Fluids, 10, 1417 (1967); J. K. Batchelor, Phys. Fluids. Suppl. II 12, 233 (1969); R. H. Kraichnan, and D. Montgomery, Rep. Prog. Phys. 43, 35 (1980).

[9] One could also perform a sequential average, scanning consecutively the time series. This is the original procedure used in the calculation of exit distances for three dimensional turbulence in [4]. It is easy to show, that the sequential procedure is equivalent to the random one after a suitable normalization [4].

[10] J. Paret, M.-C. Jullien, and P. Tabeling, Phys. Rev. Lett. 83, 3418 (1999).

[11] V. Borue, Phys. Rev. Lett. 71, 3967 (1993).

[12] P. Santangelo,R. Benzi, and B. Legras, Phys. Fluids A 1 (6), 1027 (1989).

[13] K. Nam, E. Ott, T. M. Antonsen, Jr., and P. N. Guzdar, Phys. Rev. Lett. 84, 5134 (2000);K. Nam, T. M. Antonsen, Jr., P. N. Guzdar, and E. Ott, Phys. Rev. Lett. 83, 3426 (1999).

[14] R. Benzi, S. Ciliberto, R. Tripiccione, C. Baudet, F. Massaioli, and S. Succi, Phys. Rev. E 48 R29 (1993).

[15] We refrain from giving a measurement for the relative exponent with $p=1$. This is because already in the exactly solvable case of exit distances of a twice differentiable extremum, i.e. the case of a signal like (5) with $h=2$, one can prove that for $p=1$ exit-distance moment has logarithmic corrections which strongly perturb the scaling. 